\title{
Pediatric Mechanical Support with an External Cardiac Compression Device
}

Minoo N Kavarana1*, Howard M Loree II², Robert B Stewart ${ }^{2}$, Michael T Milbocker ${ }^{3}$, Robert L Hannan ${ }^{4}$, George M Pantalos ${ }^{5}$ and Robert TV Kung ${ }^{2}$

${ }^{1}$ Department of Surgery, Medical University of South Carolina, Charleston, SC, 29425, USA

${ }^{2} A B I O M E D$, Danvers, MA, United States

${ }^{3}$ Promethean Surgical Devices, Inc., East Hartford, CT, United States

${ }^{4}$ Department of Surgery, Miami Children's Hospital, Miami, FL, United States

${ }^{5}$ Department of Surgery, University of Louisville, Louisville, KY, United States

\begin{abstract}
The PediBooster external cardiac compression device is a minimally invasive, non-blood contacting Biventricular Assist Device (BiVAD) intended for pediatric use. It is being developed as a palliative therapy for acute Postcardiotomy Shock (PCS). The PediBooster extracardiac wrap is pneumatically actuated to circumferentially compress the heart, providing co-pulsation support. Attachment is via a novel hydrogel coating. Early versions of the wrap were tested in vivo using a single ventricle congenital heart disease model with postcardiotomy shock, which proved unstable and demonstrated high peri-operative mortality. The final wrap design was tested in 4 acute studies with piglets $(5.1 \pm$ $0.3 \mathrm{~kg}$ ), where the combination of ASD and PA banding induced acute right ventricular dysfunction. Data collected included routine hemodynamic values, TEE, video of the exposed heart, and cardiac histology. The model proved stable for support durations ranging from 2 to 16 hours. The wrap restricted the heart in 3 of the 4 animals, as evidenced by increased diastolic LVP during support compared to the baseline failure condition. TEE and video data showed good attachment and function of the wrap, particularly during the final $16 \mathrm{hr}$ study. This model of congenital heart disease shows promise for chronic (24-72 hr) studies. Ventricular filling during support may be improved by adjusting wrap dimensions to eliminate end diastolic restriction.
\end{abstract}

Keywords: Pediatric mechanical support; Congenital heart disease model

\section{Introduction}

Although mechanical devices have been successfully used to support impaired ventricular function secondary to acquired cardiac pathology in adults, little has been accomplished for postoperative pediatric myocardial mechanical stabilization in children with congenital cardiac disease [1]. Conditions such as anomalous origin of the coronary artery from the pulmonary artery, critical aortic stenosis, and tetralogy of Fallot which require surgical correction may suffer from postcardiotomy myocardial failure. The need for postoperative myocardial support should therefore be anticipated after cardiac surgical repair. Single ventricle patients undergoing the Norwood procedure have a significant incidence of postoperative myocardial dysfunction and often need mechanical cardiac support. Treatment options are very limited, with ECMO being the current standard of care [2].

The objective of ECMO is to provide cardiopulmonary support for the (short to intermediate) period of myocardial dysfunction. As a method of cardiac resuscitation, ECMO is invasive, fraught with complications, and therefore expensive. Results from the ELSO registry demonstrate a $40 \%$ overall survival with only a $27 \%$ survival for neonates and single ventricle patients [3]. This report highlights the need for effective devices that can support postcardiotomy pediatric cardiac failure, especially in infants. Although univentricular assist devices may be used in select cases, most pediatric patients require biventricular support [4]. A significant number of children have right ventricular failure, making implementation of mechanical support technically challenging [5]. Hypoxemia frequently follows long, complicated corrective procedures or may be present before the initiation of ECMO (i.e. total anomalous pulmonary venous drainage with obstruction). Children do not tolerate right ventricular failure, either primary or secondary, and require right ventricle support for the subsequent elevation of central venous pressures.

At present, a few functional pediatric mechanical support devices are available. The initial medium term results with the Berlin Heart and the Medos pediatric VADS are promising, but remain invasive with relatively high bleeding and thromboembolic events [5-8].

The PediBooster is an extracardiac biventricular assist device being developed as support for acute postcardiotomy shock following congenital heart surgery. A major need is following correction of hypoplastic left heart syndrome, which has a very high use of mechanical support (e.g. ECMO) [2]. We previously demonstrated that epicardial direct cardiac compression for cardiogenic shock provides biventricular cardiac support and augmentation in an animal model of heart failure using Esmolol beta blockade [3]. This seemed to avoid a blood surface interface with its associated thromboembolic and immunologic sequelae, and could be placed rapidly with technical ease.

In order to closely mimic postcardiotomy shock after complex neonatal cardiac surgery we evaluated PediBooster support in a previously validated single ventricle model of congenital heart disease which mimics the hypoplastic left heart syndrome [9]. The complexity of this model led to the development of a simplified model of congenital

*Corresponding author: Minoo N Kavarana, Department of Surgery, Medical University of South Carolina, 96 Jonathan Lucas Street, CSB 424/MSC 613 Charleston, SC 29425, USA, Tel: 843792 3361; Fax: 843792 9783; E-mail: kavarana@musc.edu

Received March 05, 2013; Accepted March 26, 2013; Published April 04, 2013

Citation: Kavarana MN, Loree HM, Stewart RB, Milbocker MT, Hannan RL, et al. (2013) Pediatric Mechanical Support with an External Cardiac Compression Device. J Cardiovasc Dis Diagn 1: 105. doi:10.4172/2329-9517.1000105

Copyright: ( 2013 Kavarana MN, et al. This is an open-access article distributed under the terms of the Creative Commons Attribution License, which permits unrestricted use, distribution, and reproduction in any medium, provided the original author and source are credited. 
Citation: Kavarana MN, Loree HM, Stewart RB, Milbocker MT, Hannan RL, et al. (2013) Pediatric Mechanical Support with an External Cardiac Compression Device. J Cardiovasc Dis Diagn 1: 105. doi:10.4172/2329-9517.1000105

Page 2 of 6

heart disease involving acute pulmonary stenosis with an unrestrictive atrial septal defect. Results from both models are discussed.

\section{Methods}

The PediBooster is a minimally invasive, extracardiac assist device (Figure 1). The device is a pneumatically actuated, flexible device positioned to circumferentially compress the myocardium. 5 cc polyurethane balloons are incorporated into a thin polypropylene mesh. The mesh is coated with a hydrogel which maintains a reversible adhesive attachment to the myocardium. The balloons are connected to polyurethane tubes which are attached to the pneumatic driver (modified iPulse console, Abiomed, Danvers, MA). The device can be timed and synchronized with the R wave on ECG with the help of epicardial atrial pacing wires to provide co-pulsation mechanical circulatory support.

The PediBooster was tested in vivo using two congenital heart models demonstrating acute postcardiotomy shock. The studies were approved by the Institutional Animal Care and Use Committee of the University of Louisville.

\section{Single Ventricle (SV) model}

The juvenile (4-7 kg) porcine model of Kavarana et al. was initially used in five animals [9].

\section{Surgical preparation}

The piglets were anesthetized, intubated, and placed on the ventilator. A femoral arterial line was placed for monitoring and blood sampling. An internal jugular venous line was inserted for central venous pressure monitoring and intravenous access. A dual transducer pressure catheter (various models, Millar Instruments, Inc., Houston, Texas) was passed from the right carotid artery via the aorta across the valve and into the Left Ventricle (LV) for pressure monitoring and data collection. Ultrasonic flow probes (various models, Transonic Systems, Inc., Ithaca, NY) were placed around the ascending aorta and the main pulmonary artery for continuous flow measurement. Carotid artery and femoral vein cut downs were performed in preparation for the single ventricle model as previously described [9]. After median sternotomy and systemic heparinization, a modified aorto-pulmonary shunt was constructed with a $4 \mathrm{~mm}$ reinforced PTFE graft (W.L. Gore \& Associates, Inc., Flagstaff, AZ). A 5 Fr Edwards Life sciences fogarty dilation atrial septostomy catheter was then passed via the right femoral vein into the right atrium. With the help of echocardiographic guidance and digital palpation, the catheter was guided across the patent foramen ovale. A pullback septostomy was then performed, followed by tricuspid valve avulsion performed with the same catheter using multiple passes across the tricuspid valve. This was followed by gradual occlusion of the pulmonary artery and release of the shunt. Figure 2 illustrates the basic steps of the SV model, which mimics a hypoplastic left heart syndrome after the stage 1 Norwood repair. PCS occurred rapidly after conversion to SV physiology and decreased aortic flow $(\mathrm{AOF})<0.15 \mathrm{~L} / \mathrm{min}$. Following demonstration of shock, the PediBooster was placed and actuated.

Although the SV model is useful, it is known to be a highly complicated model of congenital heart disease with high mortality rates. Therefore, a need exists for a simpler model of congenital heart disease. Keeping in mind essential properties of a congenital heart model with postcardiotomy shock, which are low oxygenation and low cardiac output, we developed and evaluated a simplified alternative model of postcardiotomy shock following congenital heart surgery.

\section{Acute pulmonary stenosis with atrial septal defect (PS/ASD) model}

In order to create a more stable and reliable model of congenital heart disease, we evaluated a new concept which would combine an atrial level shunt with acute pulmonary stenosis (Figure 3 ) created by

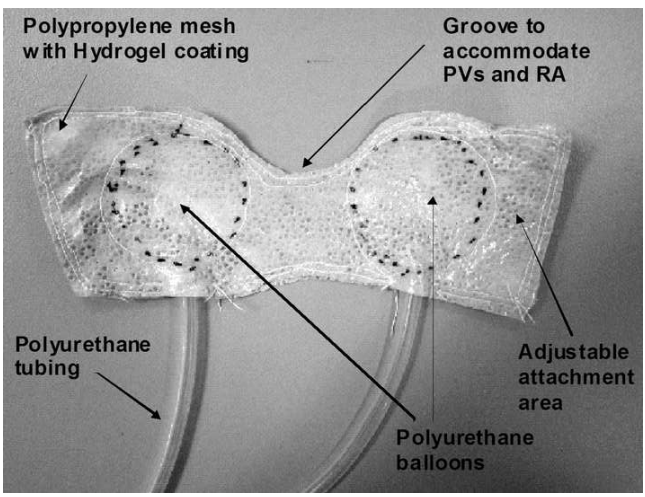

Figure 1: PediBooster extracardiac wrap design.

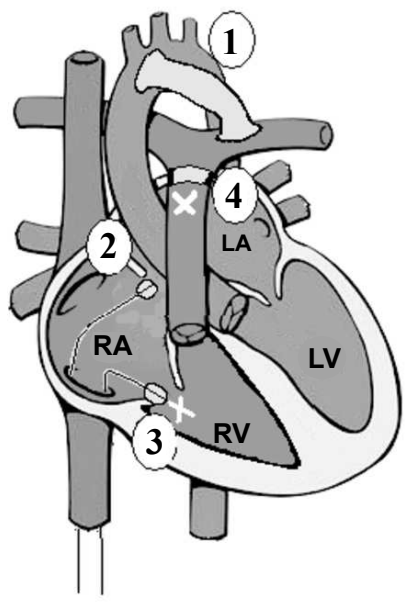

Figure 2: Single ventricle (SV) model.

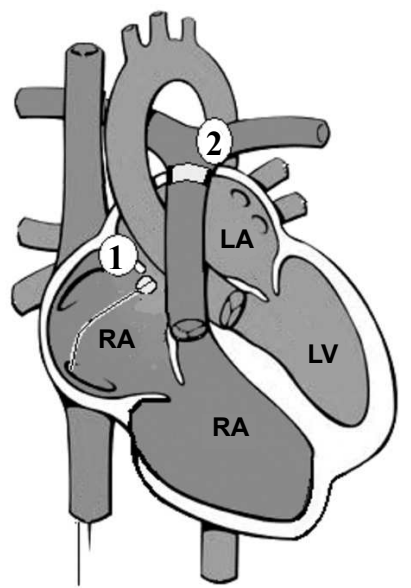

Figure 3: Acute pulmonary stenosis and atrial septal defect (PS/ASD) model. 
Citation: Kavarana MN, Loree HM, Stewart RB, Milbocker MT, Hannan RL, et al. (2013) Pediatric Mechanical Support with an External Cardiac Compression Device. J Cardiovasc Dis Diagn 1: 105. doi:10.4172/2329-9517.1000105

Page 3 of 6

a restrictive pulmonary artery band. This was modeled by creating both atrial septostomy and Pulmonary Artery (PA) banding (Figure 4). Adjusting the PA band resulted in acute Right Ventricular (RV) outflow obstruction, with a resultant increase in the right ventricular after load to initiate cardiogenic shock, while the atrial septostomy ensures venous mixing in the presence of elevated right ventricular inflow pressures. Tightening of the PA band, was followed by acute right ventricular dilatation, decreased pulmonary blood flow with resultant reduced pulmonary venous return and decreased aortic blood flow.

\section{Surgical preparation}

Using a 4-7 kg pig, an acute PS/ASD conversion was completed in four animals. After appropriate anesthesia and sedation, the animal was intubated. Central lines for Central Venous Pressure (CVP) monitoring and intravenous access were placed in the left internal jugular vein and left femoral vein. An arterial pressure line was placed in the left femoral artery. A dual transducer Millar pressure catheter was inserted via the right internal carotid artery, aorta, across the aortic valve, and placed in the LV. Transonic Systems ultrasonic flow probes were placed around the ascending aorta and the main pulmonary artery for continuous flow measurement. A balloon septostomy catheter was then introduced through the right femoral vein into the right atrium. Echocardiographic guidance and manual palpation helped guide the catheter across the patent foramen ovale. The balloon was inflated and withdrawn across the septum to create an unrestricted ASD. Oximetry $\left(\mathrm{satO}_{2}\right), \mathrm{PA}$ flows (PAF), and $\mathrm{AOF}$ were monitored. Flows and sat $\mathrm{O}_{2}$ decreased after the conversion. At 20 minutes of $\mathrm{HF}$ (defined as $\mathrm{PAF}$ and AOF $<50 \%$ preop baseline, with sat $\left.\mathrm{O}_{2}<60 \%\right)$ cardiac arrest occurred if support was not initiated or PA restriction was continued. Once support was initiated, PA banding was resumed to avoid excessive left-to-right shunt.

Direct cardiac compression with the PediBooster was performed to provide augmentation initially in four acute studies. The device was attached to the epicardial surface of the heart via a novel hydrogel coating. Left ventricular pressure-flow curves and relationships were evaluated in addition to various hemodynamic and hematologic outcome variables including aortic and pulmonary flows, LV and aortic pressures, lactic acid levels, arterial blood gases, and plasma free hemoglobin. Trans-esophageal echocardiography was used in a few studies to assess the fit and effects of external cardiac compression. After termination, the hearts were examined macroscopically and microscopically for evidence of trauma.

\section{Results}

\section{SV model}

Single ventricle conversion resulted in a reduction of mean aortic flow to $40-50 \%$ of the baseline with severe ventricular dysfunction. Under PediBooster support, AOF of $60-75 \%$ of baseline was obtained for the SV HF model, although these results were not stable in most cases. Support was continued for up to 2 hours in five animals, without any gross signs of cardiac contusions/trauma from extracardiac compression. Despite the enhanced AOF, oxygenation remained low; which may have resulted from partial thrombosis of the aorticto-pulmonary artery shunt used in the model. The PediBooster also provided $>0.50 \mathrm{~L} / \mathrm{min}$ flow for both an asystolic and a fibrillating heart $(\mathrm{AOF}<0.05 \mathrm{~L} / \mathrm{min})$. Figure 5 demonstrates baseline aortic and LV pressure significantly reduced with the single ventricle model followed by adequate augmentation to $60-75 \%$ of baseline pressure with PediBooster support. Figure 6 demonstrates aortic flows after ventricular fibrillation that occurred following a SV model completion, which was followed by PediBooster support. This shows adequate augmentation even after fibrillatory arrest. There was a substantial reduction in LV pressure (LVP), Aortic Pressure (AOP), and AOF from baseline after SV model completion. PediBooster support restored LVP, AOP, and AOF to approximately $75 \%$ of baseline from failure parameters both in 1:1 and 1:2 modes.

We calculated hemodynamic augmentation in order to assess the efficacy of the device. We observed a significant reduction in contractility with the SV model, and an improvement in contractility with the device.

\section{PS/ASD model}

Sustained heart failure was induced by restricting the mean PA flow by nearly $50 \%$ via PA banding. This resulted in a reduction of oxygen saturation from normal (98-99\%) to hypoxic (around $80 \%$ ). Figures 7 demonstrate a sustained reduction in AOP, LVP, AOF, and PAF after the acute PS/ASD model (Vertical lines in the figures indicate interrupted recordings.) Figure 8 shows the restoration of aortic and LV pressure back to normal after PediBooster support.

The PS/ASD model proved stable for support durations ranging from 2 to 16 hours. Without support, the model led to complete heart failure within 20 minutes. The wrap restricted the heart in three of the four animals, as evidenced by increased diastolic LVP, leading to

AOP/LVP vs. Time

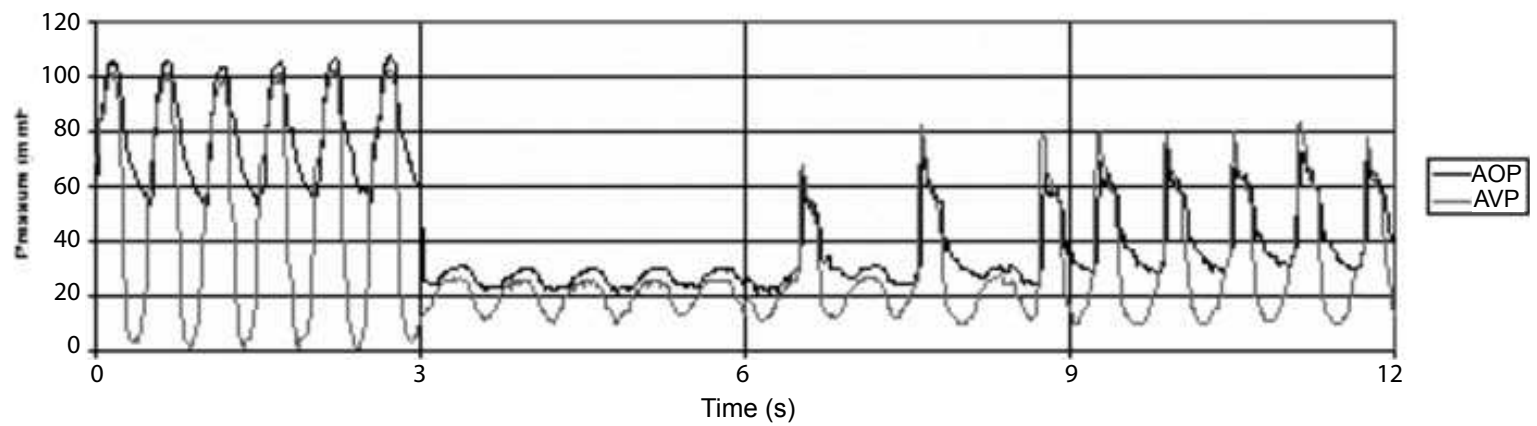

Figure 4: AOP and LVP at baseline $(0<\mathrm{t}<3 \mathrm{~s})$, after initiating SVM $(3<\mathrm{t}<6 \mathrm{~s})$, and after initiating PediBooster support $(6<\mathrm{t}<9 \mathrm{~s}$ for $1: 2$ support; $9<\mathrm{t}<12 \mathrm{~s}$ for 1:1 support). 
Citation: Kavarana MN, Loree HM, Stewart RB, Milbocker MT, Hannan RL, et al. (2013) Pediatric Mechanical Support with an External Cardiac Compression Device. J Cardiovasc Dis Diagn 1: 105. doi:10.4172/2329-9517.1000105

Flow VS. Time

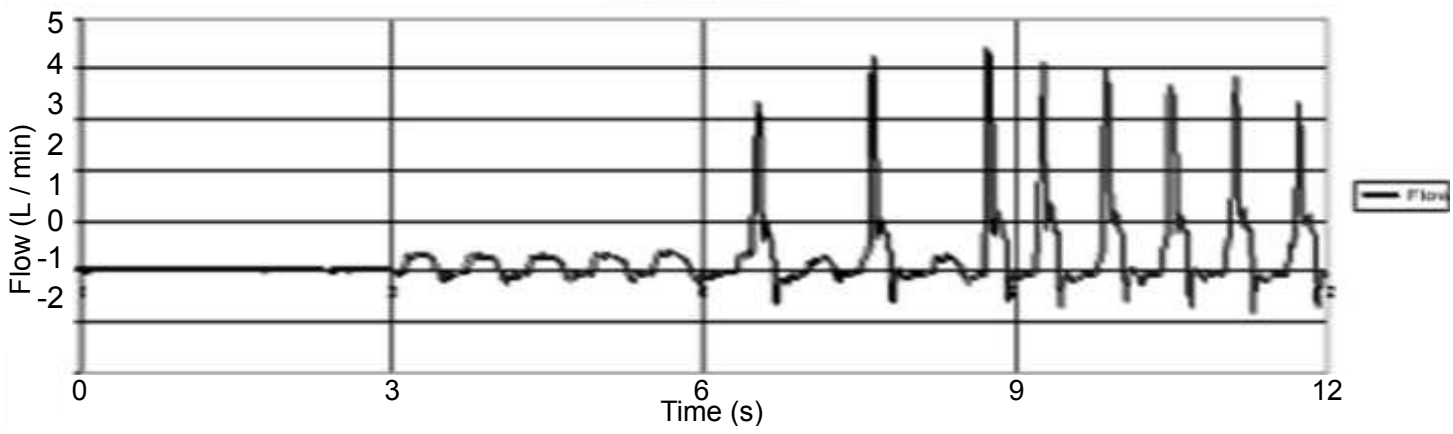

Figure 5: AOF during post-SV model fibrillation without $(0<t<6 \mathrm{~s})$ and with PediBooster support $(6<t<12 \mathrm{~s}$ with 1:2 followed by 1:1 support)

LV/AO Flow vs. Time

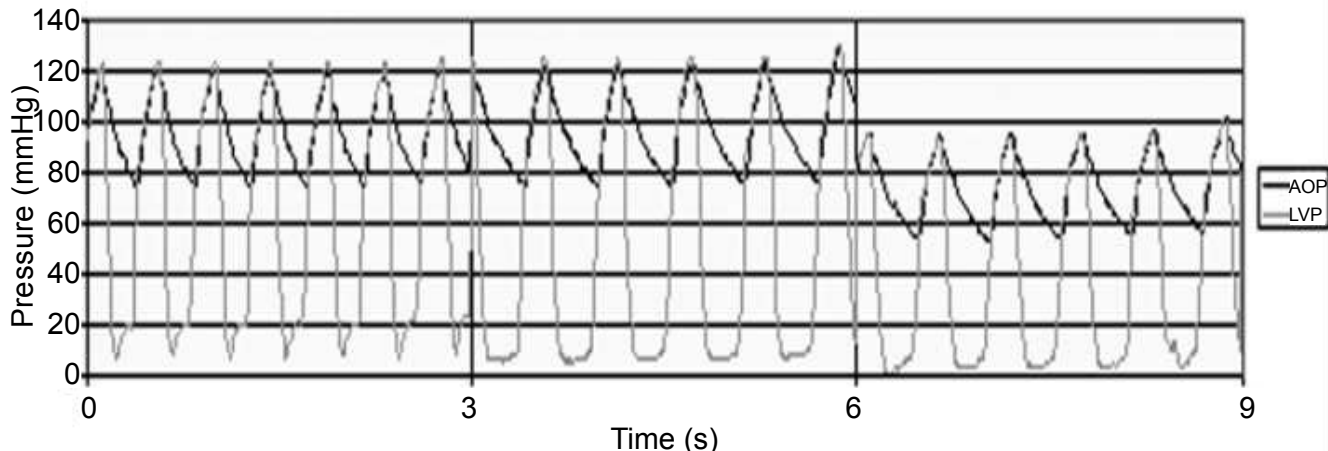

Figure 6: AOP and LVP at baseline $(0<t<6 \mathrm{~s})$ and after initiating acute PS/ASD model $(6<t<9 \mathrm{~s})$ showing stable failure prior to initiating PediBooster support.

AO/PA Flow vs. Time

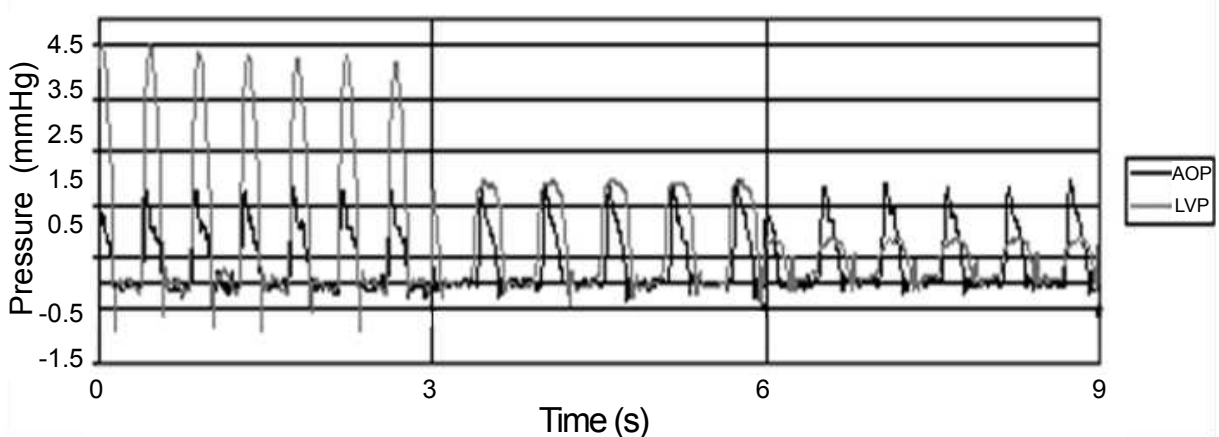

Figure 7: AOF and PAF at baseline $(0<t<3 \mathrm{~s})$ and after initiating acute PS /ASD model $(3<\mathrm{t}<6 \mathrm{~s})$ showing stable failure $(6<\mathrm{t}<9 \mathrm{~s})$ prior to initiating PediBooster support.

decreasing AOF, and PAF eventually reverting back to the baseline failure condition and the end of support. Table 1 summarizes the results of the PS/ASD model.

TEE and video data showed good attachment and function of the wrap, particularly during the final $16 \mathrm{hr}$ study. The summary results given in table 1 are averages over both times for a single experiment which are then average over the studies. The lower flows and sat $\mathrm{O}_{2}$ during support compared to the short failure duration resulted primarily from the downward trend of these parameters over time during any one experiment.

After removing the wrap, the hydrogel attaching the PediBooster wrap to the epicardium was easily separated by finger tip dissection to expose the heart. There was no damage to the epicardium as the hydrogel was dissected with some hydrogel remaining adhered to the epicardial surface. There was obvious visible injury to the epicardial surface in the form of small areas of bruising on the surface of the right 
LV/AO Pressure vs. Time

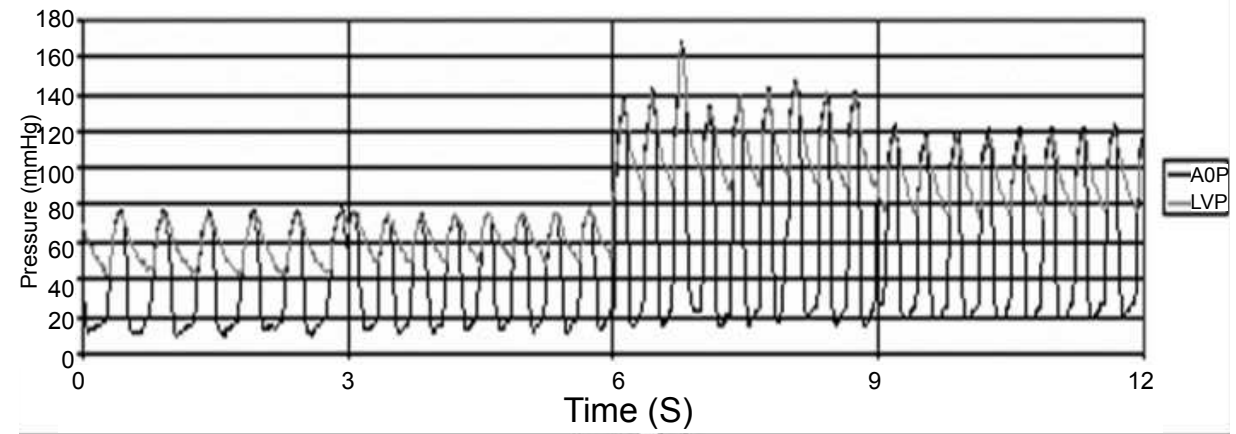

Figure 8: Aortic and LV pressure after initiating acute PS/ASD model $(0<t<6 \mathrm{~s})$ and after PediBooster augmentation $(6<\mathrm{t}<12 \mathrm{~s})$.

\begin{tabular}{|c|c|c|c|}
\hline & \multicolumn{3}{|c|}{ PediBooster In vivo Hemodynamic Data $(\mathrm{n}=4$, mean $\pm \mathrm{SD})$} \\
\hline Measurement & Healthy & Failure & $68 / 7 \pm 34 / 2$ \\
\hline LVP $(\mathrm{mmHg})$ & $105 / 8 \pm 15 / 3$ & $0.35 \pm 0.18$ & $109 / 15 \pm 48 / 8$ \\
\hline AOF $(\mathrm{L} / \mathrm{min})$ & $0.54 \pm 0.21$ & $0.42 \pm 0.32$ & $0.25 \pm 0.18$ \\
\hline PAF $(\mathrm{L} / \mathrm{min})$ & $0.69 \pm 0.18$ & $79 \pm 6$ & $74 \pm 0.30$ \\
\hline $\operatorname{satO}_{2}(\%)$ & $98 \pm 2$ & $9 \pm 5$ \\
\hline CVP $(\mathrm{mmHg})$ & $5 \pm 2$ & $16 \pm 4$ \\
\hline
\end{tabular}

Table 1: PediBooster support from stable failure with acute PS/ASD model.

and left ventricle. Upon opening the heart, there was no intraventricular thrombus present. However, small portions of the right ventricular wall appear bruised through the entire thickness.

\section{Device limitations}

Diastolic restriction is a device limitation well known to occur with external cardiac compression which can be significantly altered with adjustment of device fit. Myocardial bruising which we observed in our autopsy studies was mostly epicardial with a small area which was transmural. Further investigation of fit adjustment and deviceepicardial interaction will cast light on these findings and help guide future device modifications. In comparison with ECMO, direct cardiac compression does not have the same salutary effect on hypoxemia but may provide some improvement through improved pulmonary blood flow. The hydrogel epicardial surface interaction which seems favorable in these short term studies will need further investigation in future chronic studies.

\section{Discussion}

Children undergoing repair of complex congenital heart defects have a recognized incidence of postcardiotomy cardiogenic shock requiring mechanic cardiac support $[10,11]$. The dearth of devices for pediatric use and the invasiveness with associated bleeding and thromboembolic complications stimulated the development of the PediBooster for this indication.

The PediBooster was initially evaluated on a previously validated single ventricle model of congenital heart disease which closely mimics the post-Norwood repair physiology in patients with the hypoplastic left heart syndrome. In view of the high complexity and inherent instability of this single ventricle model initially used, we developed a simpler and more stable model of congenital heart disease.

With this revised model, we were able to demonstrate more stable and reproducible hypoxic cardiac failure with our model of pulmonary stenosis and unrestricted ASD. Although the SV model is an excellent
HF model, it is technically very challenging for evaluation of longer term support. The acute PS/ASD model approximates hemodynamics and physiology associated with acute neonatal HF, making it a useful model to test pediatric cardiopulmonary support devices.

We demonstrated good pressure and flow maintenance with the PediBooster after having established severe acute and chronic heart failure with hypoxia. We did observe systolic augmentation, which was limited by restriction of diastolic filling. This diastolic restriction resulted in a gradual decline of flow during the support duration. There was histologic evidence of epicardial bruising with some myocardial injury over both ventricles. These observations suggest that the device fit should err on the loose side so that diastole is not impeded. Concurrently, systolic inflation should be controlled or limited to avoid complete emptying of the ventricles, thus avoiding myocardial bruising over extended support.

\section{Conclusion}

These studies therefore demonstrate the feasibility of short term SV support using the PediBooster, while indicating the need to further examine ways to eliminate the diastolic restriction and myocardial damage observed after extended PediBooster support.

Further evaluation of longer duration of support with the device along with less invasive insertion and removal techniques would help the transition of this device to clinical use.

\section{Acknowledgment}

This research was funded by NIH NHLBI SBIR grant 5R44HL073552-03.

\section{References}

1. Pennington DG, Swartz MT (1993) Circulatory support in infants and children Ann Thorac Surg 55: 233-237.

2. Allan CK, Thiagarajan RR, del Nido PJ, Roth SJ, Almodovar MC, et al. (2007) Indication for initiation of mechanical circulatory support impacts survival of infants with shunted single-ventricle circulation supported with extracorporeal membrane oxygenation. J Thorac Cardiovasc Surg 133: 660-667. 
Citation: Kavarana MN, Loree HM, Stewart RB, Milbocker MT, Hannan RL, et al. (2013) Pediatric Mechanical Support with an External Cardiac Compression Device. J Cardiovasc Dis Diagn 1: 105. doi:10.4172/2329-9517.1000105

3. del Nido PJ (1996) Extracorporeal membrane oxygenation for cardiac support in children. Ann Thorac Surg 61: 336-339

4. Black MD, Coles JG, Williams WG, Rebeyka IM, Trusler GA, et al. (1995) Determinants of success in pediatric cardiac patients undergoing extracorporeal membrane oxygenation. Ann Thorac Surg 60: 133-138.

5. Rockett SR, Bryant JC, Morrow WR, Frazier EA, Fiser WP, et al. (2008) Preliminary single center North American experience with the Berlin Heart pediatric EXCOR device. ASAIO J 54: 479-482.

6. Arabía FA, Tsau PH, Smith RG, Nolan PE, Paramesh V, et al. (2006) Pediatric bridge to heart transplantation: application of the Berlin Heart, Medos and Thoratec ventricular assist devices. J Heart Lung Transplant 25: 16-21.

7. Hetzer R, Potapov EV, Stiller B, Weng Y, Hübler M, et al. (2006) Improvement in survival after mechanical circulatory support with pneumatic pulsatile ventricular assist devices in pediatric patients. Ann Thorac Surg 82: 917-924.
8. Agati S, Ciccarello G, Ocello S, Salvo D, Marcelletti C, et al. (2006) Pulsatile ECMO and VAD: a dual use of a new device in pediatric cardiac patients. ASAIO J 52: 501-504.

9. Kavarana MN, Helman DN, Williams MR, Barbone A, Sanchez JA, et al. (2001) Circulatory support with a direct cardiac compression device: A less invasive approach with the AbioBooster device. J Thorac Cardiovasc Surg 122: 786787.

10. Randsbaek F, Riordan CJ, Storey JH, Montgomery WD, Santamore WP et al. (1996) Animal model of the univentricular heart and single ventricular physiology. J Invest Surg 9: 375-384.

11. Cooper DS, Jacobs JP, Moore L, Stock A, Gaynor JW, et al. (2007) Cardiac extracorporeal life support: state of the art in 2007. Cardiol Young 2: 104-115. 